\title{
Ultrasonography in the Early Diagnosis of Diabetic Lumbosacral Radiculoplexus Neuropathy
}

\author{
Jae Young $\mathrm{An}^{\mathrm{a}}$ \\ Dae Woong Bae ${ }^{a}$ \\ Francis O. Walker ${ }^{\mathrm{b}}$ \\ ${ }^{a}$ Department of Neurology, \\ St. Vincent Hospital, College of Medicine, \\ The Catholic University of Korea, \\ Suwon, Korea \\ ${ }^{b}$ Department of Neurology, \\ Wake Forest School of Medicine, \\ Medical Center Blvd, Winston-Salem, \\ NC, USA
}

Dear Editor,

Diabetic lumbosacral radiculoplexus neuropathy (DLRPN) is an uncommon but distinct condition characterized by asymmetrical proximal lower extremity pain, weakness, and atrophy that occurs in patients with diabetes mellitus. ${ }^{1}$ Although this syndrome is a monophasic illness, patients may experience prolonged morbidity from pain and weakness, and progress to being wheelchair dependent. ${ }^{2}$ To our knowledge, this is the first report of ultrasonographic findings in DLRPN.

An otherwise healthy 59-year-old woman with mild diabetes mellitus that was managed with diet developed acute severe burning pain in the anterior left thigh. Three days later she noted weakness in her left leg, and over the course of 1 week became unable to walk unassisted. She had not recently experienced any significant weight loss. A clinical examination performed 2 weeks after the onset showed selective weakness of the muscles innervated by the left femoral and obturator nerves [hip flexion, Medical Research Council (MRC) grade I: hip adduction, MRC grade II: knee extension, MRC grade 0] and decreased sensation in the left L3-L4 dermatome. The left knee jerk was absent but her ankle jerk was normal. Laboratory tests including complete blood cell count, thyroid function, vitamin $\mathrm{B}_{12}$, folate, and autoimmune studies produced negative findings. Blood glucose was $281 \mathrm{mg} / \mathrm{dL}$ and hemoglobin A1C was 12.6\%. The erythrocyte sedimentation rate was $25 \mathrm{~mm} / \mathrm{h}$ (normal range, $0-20 \mathrm{~mm} / \mathrm{h}$ ) and her cerebrospinal fluid was normal except for elevated protein (67 $\mathrm{mg} / \mathrm{dL}$ ). Nerve conduction studies showed a reduced compound muscle action potential of the left femoral nerve, but all other findings were normal. Needle electromyography showed only increased insertional activity in the left iliopsoas muscle and no voluntary activity in muscles innervated by the femoral and obturator nerves. Follow-up testing performed 5 weeks after the onset showed no responses to stimulation in the left femoral, left saphenous, and left lateral femoral cutaneous nerves, and EMG revealed fibrillation potentials in the left psoas, vastus lateralis, adductor longus, and lumbar paraspinal muscles.

Ultrasonography (ACUSON S1000, Siemens Medical Solutions, Malvern, PA, USA) with a 5-14 MHz linear transducer performed 2 weeks after the onset of clinical symptoms revealed significantly increased cross-sectional areas of the left femoral $\left(53 \mathrm{~mm}^{2}\right)$ (Fig. 1A) and lateral femoral cutaneous $\left(5 \mathrm{~mm}^{2}\right)$ (Fig. 1C) nerves; these were more than twice the sizes of those on the contralateral side (Fig. 1B and D). We did not obtain long-axis images of these nerves, but we observed the short-axis view of the femoral nerve from the inguinal crease to the point where the femoral nerve branches, which identified swelling of the cross-sectional area of the femoral nerve over this segment. Ultrasonography revealed no significant changes in the vascularity or echogenicity of these nerves. Magnetic resonance imaging (MRI) showed perineural enhancement along the left L3-L4 nerve roots, and this enhancement-which is suggestive of inflammation-appeared to extend into the posterior aspect of the left psoas (Fig. 1E, F, and G). We diagnosed her with DLRPN and started treatment

@ This is an Open Access article distributed under the terms of the Creative Commons Attribution Non-Commercial License (https://creativecommons.org/licenses/by-nc/4.0) which permits unrestricted non-commercial use, distribution, and reproduction in any medium, provided the original work is properly cited. 

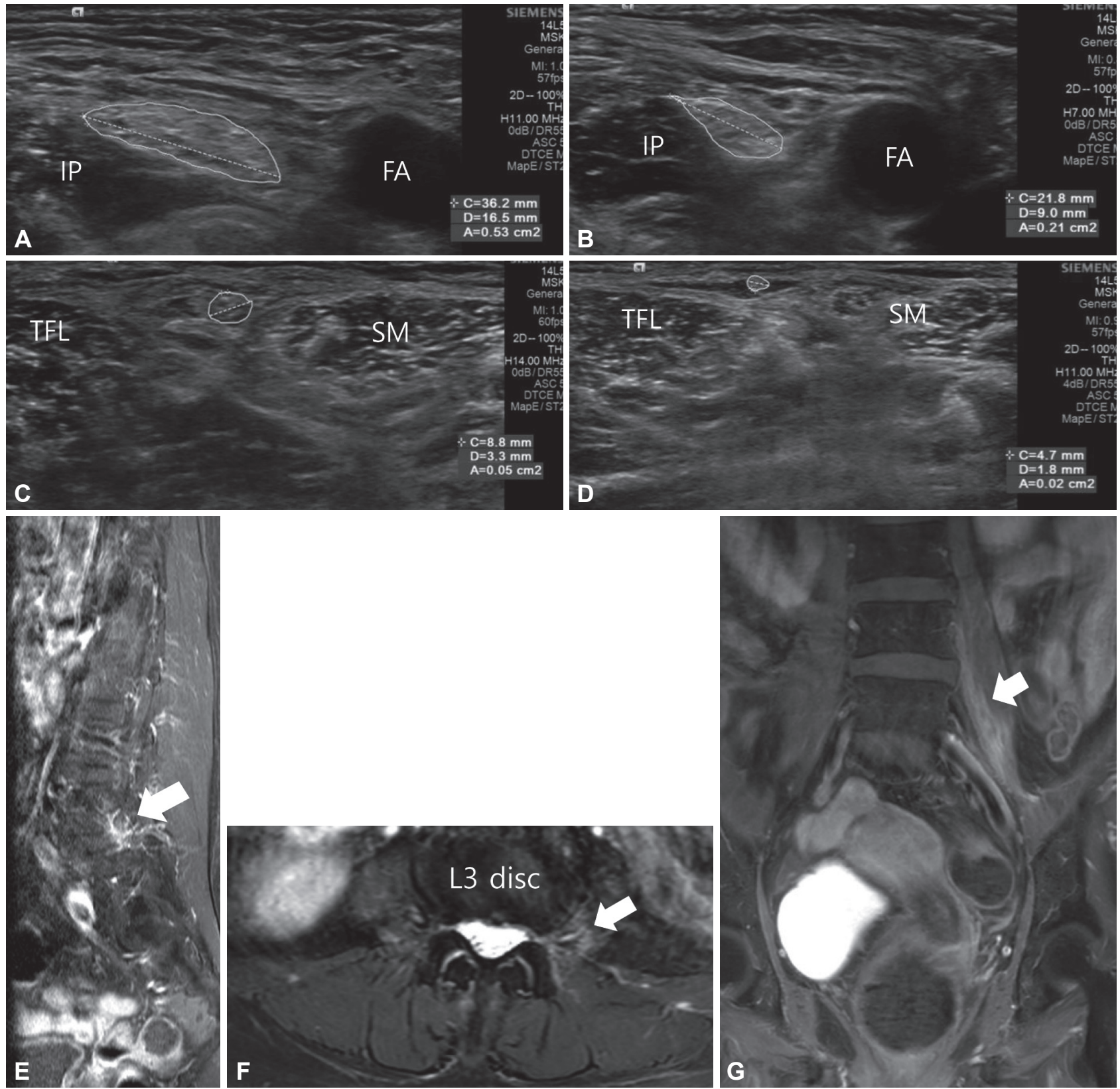

Fig. 1. Ultrasonography images showing the increased cross-sectional areas of the left femoral (A) and left lateral femoral cutaneous (C) nerves compared to the right side $(B, D)$. MRI shows perineural enhancement along the L4 root (arrow) in a T1-weighted enhanced image (E), increased signal intensity in muscle (arrow) adjacent to the L3 root in an axial T2-weighted fat-suppressed image (F), and an enhanced posterior aspect of the psoas (arrow) in a coronal T1-weighted fat-suppressed enhanced image (G). FA: femoral artery, IP: iliopsoas, SM: sartorius, TFL: tensor fascia lata.

with intravenous high-dose corticosteroid.

DLRPN involves the spinal roots, lumbosacral plexus, and peripheral nerves, and frequently affects distal as well as proximal lower limbs. One of the challenges of DLRPN is that its diagnosis is often delayed and sometimes surgery for suspected lumbar spine or disc disease is performed before the condition is correctly identified. ${ }^{2}$ There is recent evidence that DLRPN is caused by acute local microvasculitis of the spinal roots, lumbosacral plexus, and peripheral nerves, and that early treatment with steroids, immunosuppressants, or intravenous immunoglobulin can produce good outcomes. ${ }^{2}$ The severity of this disorder makes it necessary to develop improved techniques for its early diagnosis. Previous studies have shown that MRI neurography is informative for diagnosing lumbosacral radiculoplexus neuropathy., ${ }^{3,4}$ However, MRI is not always feasible due to expense, availability, or patient-related factors such as claustrophobia or the presence of metal implants. A few reports on other forms of systemic or region- 
al vasculitis have suggested that ultrasonography can reveal nerve enlargement and hyperechogenic epineurium..$^{5-7}$ Ultrasonography is a relatively new imaging modality that has not been applied previously to DLRPN.

However, a limitation of this study is that ultrasound cannot be used to image deep portions of the lumbosacral plexus, and more-extensive observations of distal motor and sensory branches were not performed. This case demonstrates the potential value of neuroimaging in patients with DLRPN. Although based on only a single case, the present findings may help guide the development of future diagnostic algorithms aimed at the early diagnosis of DLRPN.

\section{Conflicts of Interest}

The authors have no potential conflicts of interest to disclose.

\section{REFERENCES}

1. Laughlin RS, Dyck PJ. Diabetic radiculoplexus neuropathies. Handb
Clin Neurol 2014;126:45-52.

2. Dyck PJ, Windebank AJ. Diabetic and nondiabetic lumbosacral radiculoplexus neuropathies: new insights into pathophysiology and treatment. Muscle Nerve 2002;25:477-491.

3. Filosto M, Pari E, Cotelli M, Todeschini A, Vielmi V, Rinaldi F, et al. MR neurography in diagnosing nondiabetic lumbosacral radiculoplexus neuropathy. J Neuroimaging 2013;23:543-544.

4. Massie R, Mauermann ML, Staff NP, Amrami KK, Mandrekar JN, Dyck PJ, et al. Diabetic cervical radiculoplexus neuropathy: a distinct syndrome expanding the spectrum of diabetic radiculoplexus neuropathies. Brain 2012;135:3074-3088.

5. Grimm A, Décard BF, Bischof A, Axer H. Ultrasound of the peripheral nerves in systemic vasculitic neuropathies. J Neurol Sci 2014;347: 44-49.

6. Ito T, Kijima M, Watanabe T, Sakuta M, Nishiyama K. Ultrasonography of the tibial nerve in vasculitic neuropathy. Muscle Nerve 2007;35: 379-382.

7. Leupold D, Felbecker A, Tettenborn B, Hundsberger T. Nerve ultrasound as a decisive tool in nonsystemic vasculitic neuropathy: a case report. Case Rep Neurol 2016;8:108-114. 